\title{
Using Agile Methods for Infrastructure Projects: A Practical Experience
}

\author{
C.J. Torrecilla-Salinas, J. Sedeño, M.J. Escalona,and M. Mejías
}

\begin{abstract}
Agile approaches have emerged in Software Development projects during the last decade becoming a real alternative for organizations developing software. These approaches have been used successfully in several and different projects: from small teams of in-site developers to teams comprising hundreds of developers all over the world. Nevertheless, Agile approaches are not only suitable for Software Development projects, but these methodologies can also be considered to be a framework to plan, estimate and manage any kind of project s focused on quickresponse, adaptation to customers' needs and early delivery of value to costumers. This paper presents the result of an experience dealing with using an Agile framework, based on Scrum, in an infrastructure project applied to a Spanish Public Administration. According to the results of the project, the paper takes out the main lessons learned and proposes further lines of research.
\end{abstract}

Keywords Agile methodologies $\bullet$ Scrum $\bullet$ e-Government $\bullet$ Public Administration - Infrastructure

\subsection{Introduction}

Agile methodologies are becoming a solid alternative in the field of software development $[1,2]$, as they provide support to some special needs required by these kind of projects, like reduction of "time-to-market," adaptability and quick response to

C.J. Torrecilla-Salinas $(\bowtie) \bullet$ M.J. Escalona・ M. Mejías

DepartmentofComputerLanguagesandSystems ，University of Seville, Seville, Spain e-mail: carlos.torrecilla@iwt2.org; mjescalona@us.es; risoto@us.es

J. Sedeño

DepartmentofComputerLanguagesandSystems ，University of Seville, Seville, Spain

AgenciaAndaluzadeInstitucionesCulturales.JuntadeAndalucía , Sevilla, Spain

e-mail: jorge.sedeno@juntadeandalucia.es 
changes [3, 4]. However, some of the Agile approaches, like Scrum, can be considered to be a framework to support project management rather than a software development methodology [5]. This fact enables thinking that Agile approaches can be used to handle any kind of project $s$, whenever their needs can relate to quick adaptation to changing needs and early delivery of value. Normally, the work carried out by an IT department is not only associated with software development projects, but also with technology infrastructure projects, among others, therefore the feasibility of expanding the Agile approach beyond software development can allow standardizing practices and processes among these departments, by reducing effort and cost.

Based on the foregoing, this work presents the result of applying a Scrum-based Agile approach to support the processes of estimating, planning and managing an infrastructure project developed by a Spanish Public Administration, with the following objectives: assess the feasibility of using Agile approaches in IT projects unrelated to software development, with a special focus on the project management aspects and take out the main lessons learned with the project, in order to identify further lines of research.

This paper is organized into the following sections. After this introduction, Sect. 37.2 offers an overview of the related work and the proposed Agile approach and Sect. 37.3 presents the project and its environment. Then, Sect. 37.4 states the results of the project and lastly, Sect. 37.5 identifies the main lessons learned and proposes possible future lines of work.

\subsection{An Overview of the Agile Framework}

This section presents an overview of the related work and the Agile framework used during the project. The approach was based on Scrum and included elements of Agile estimating, planning and project management techniques. Figure 37.1 summarizes it as follows:

\subsubsection{Related Work}

Although the majority of research in the field of Agile is focusing on software development projects, there are also approaches trying to use Agile approaches in other fields of System Development. For instance, there are approaches centered on

Fig. 37.1 Agile framework

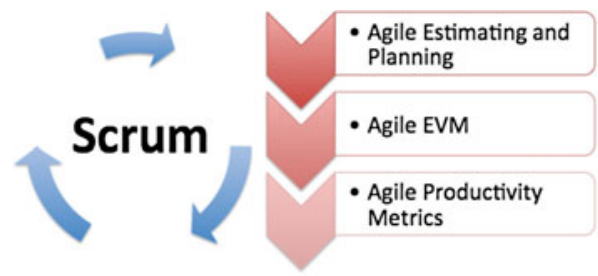


the use of Agile techniques in hardware development projects included in the so called Agile System Engineering [6], but they are more focused on assessing whether Agile approaches are suitable enough for developing hardware systems than on describing the details of the management process in that project. There are also proposals regarding the use of Agile approaches for rapid hardware modeling [7], but this kind of projects are more related to the use of software to model hardware than to the assessment of Agile in non-software projects. In addition, there are other approaches dealing with the use of Agile approaches in infrastructure projects, as the one proposed by Debois [8], which describes some case studies and takes out patterns concerning the introduction of Agile in this type of projects. However, this work is not considering aspects such as how the projects are estimated, planned and managed. In our work, we present an agnostic framework suitable for different types of projects covering elements such as project estimation, project plan, project management and productivity improvement, and assess it in a non-software development project with the aim of extracting the lessons learned.

\subsubsection{Scrum}

In 2001, a work known as the "Agile manifesto" [9] was published including the main principles and values characterizing Agile approaches. One of the most popular Agile methodologies is Scrum [4], proposed by Jeff Sutherland and Ken Schwaber [10]. It is a framework that supports product development [5] founded on an iterative and incremental approach for project management. Scrum proposes a development process divided into Sprints, time-boxed periods lasting 2-4 weeks that will be repeated during the project. The Scrum process uses an artifact called Product Backlog, containing all the features to develop during the project, ordered by priority. The team selects the features to develop and commits to it at the beginning of each Sprint, creating what is called the Sprint Backlog. The progress is checked daily once the Sprint is planned. The resultant work is reviewed in liaison with the main stakeholders after each Sprint in a Sprint demo and the development process is reviewed in a Sprint retrospective.

\subsubsection{Agile Estimating and Planning Techniques}

Scrum does not define any technique to establish and estimate the pieces of functionality that are part of the Product Backlog. Thus, some other Agile techniques are normally used to fill these gaps. In this particular case, the features included in the Product Backlog are "user stories" [11, 12]. Two techniques will be used to estimate user stories. On one hand, the development team will calculate the size of each story by means of an ideal unit called "story points" [13]. The "Planning poker" technique will be used to perform estimates $[13,14]$. On the other hand, the value of 
each story will also be estimated by means of the "Value point analysis" technique [15]. For this value, the customers or their representatives should perform the estimation. With these two values, we can obtain a relation between the cost and benefit of every user story (it is called "Return of investment" or ROI) by dividing the value in value points by the size in story points. This magnitude can be used to order the Product Backlog and establish its priority. The process of estimating and planning will be constant during the project, reserving a slot of time to review the Product Backlog content during each Sprint. This process, known as Product Backlog Grooming [16], can take up to $10 \%$ of the available time on each Sprint.

\subsubsection{Agile Earned Value Metrics}

An Agile-based approach to the Earned Value Management techniques [17] can be used in order to control the planned schedule and cost the project entails. These techniques deal with measuring the relationship among cost, scope and schedule along the project [18], based on calculating two main indexes, the Cost Performance Index (CPI), which measures the relation between estimated and real cost, and the Schedule Performance Index (SPI), which measures the relation between estimated and real schedule. At the end of each Sprint, the proposed Agile approach to EVM measures these indexes to calculate them, through both, the estimated and completed story points and the estimated and dedicated work hours.

\subsubsection{Agile Productivity Metrics}

A number of productivity metrics will be used to facilitate the team's performance process of continuous improvement. They will be obtained by means of planned and finished story points and dedicated work hours, with the aim of keeping a lightweight process. These data are also obtained through the EVM calculation. Metrics were proposed by Downey and Sutherland [19] and they are calculated per Sprint. The following ones will be used:

- Team Velocity (in Hours): It calculates the product of the velocity in story points by the team's average amount of hours per story point.

- Work Capacity: It deals with the total amount of hours worked by the team during a Sprint.

- Focus Factor: It refers to the result of dividing the Team Velocity (in Hours) by the Work Capacity.

- Percentage of Accepted Work: It is the result of dividing the hours dedicated to accepted work by the Work Capacity of the team during a Sprint.

- Target Value Increase (TVI+): It refers to the finished story points of certain Sprint divided by the average story points of all finished Sprints. 


\subsection{The Project and Its Environment}

This section presents the environment in which the project was developed, the project itself and its main results, which will be discussed in the following sections.

\subsubsection{The Environment: The Regional Government of Andalusia}

The project presented in this paper was developed by the regional government of Andalusia, a region located in the south of Spain. Junta de Andaluc'a is the name of Andalusia's regional government; counting with more than 200,000 employees, it is one of the main economic actors in the region [20]. This project was developed by one of the ministries of the Junta de Andaluc'a: the regional Ministry of Culture and Sport, which is in charge of developing and coordinating public policies both in the cultural and sports areas. Some of its duties are related to the management of public museums, archives, sports clubs, art galleries and theaters. It is also responsible for supporting regional cultural and sports industries, together with other public and private stakeholders. The ICT Department, one of the departments of the Ministry of Culture and Sport, is in charge of this project, being responsible for all Information Technology policies in the Ministry. It is mainly responsible for providing the entire ICT infrastructure needed to operate all Ministry internal systems.

It is important to highlight that, as in the last years citizens' demands on quick and easy access to public services have increased, governments have launched different initiatives to cover these needs, such as e-Government public services $[21,22]$. Due to the commitment of the regional Ministry on the e-Government strategy, the number of systems under the responsibility of its ICT Department has increased dramatically.

\subsubsection{The TOPOS Project}

As it was mentioned, the ICT Department of the regional Ministry of Culture and Sport is in charge of providing the entire ICT infrastructure that supports all the Ministry systems. On the one hand, in the last years, the number of systems under the responsibility of the Ministry highly increased due to the e-Government strategy of both the regional and the national government. On the other hand, the effects of today's economic crisis made the organization increase the efforts on costs rationalization and optimization. These two main reasons made the ICT Department of the Ministry start with a project to improve its internal infrastructure and provide a better service with fewer resources. The project was called TOPOS and it lasted more than 
a year, starting on January 2012 and finishing on February 2013. The main goals of the project were:

- Reorganize the environments where the systems were deployed, including a development, pre-production and production environment for each system, using virtualization solutions. It intended to reduce the number of physical servers, in order to save space and energy consumption on the datacenter.

- Uniform the version of the software products used to support the systems (operative systems, databases, application servers...), in order to reduce the maintenance costs required to run these systems.

- Update the versions of some homemade software products that support the general systems of the Ministry, in order to eliminate some errors and security risks.

- Clean and decommission some obsolete systems, in order to clean and free resources that could be used to support new services.

- Get high availability configuration of all e-Government services operating into the regional Ministry to improve the service offered to citizens.

As TOPOS is an infrastructure project, it is affected by the business projects of the regional Ministry and their different priorities, whereby to be able to respond to possible changes on requirements and priorities, an Agile approach was established, with Scrum as a base framework.

A team of five members, partially working on the project and coming from both the system management team and the e-Government bureau of the ICT Department, developed the project by means of internal resources. Then, the following roles were played among the members of the team:

- A Scrum Master, whose goal was to ensure the adherence of the team to Scrum practices and help it identify and remove detected errors.

- A Product Owner, who tried to identify the business needs and prioritize them using the Product Backlog.

- A Development Team, who aimed to develop each of the requested features during the selected Sprint.

It has to be mentioned that a particular aspect of TOPOS project, as it concerned the basic infrastructure of the regional Ministry, deals with its impact both on the internal and external users (mainly citizens), for they will be affected, for instance, by services stops when performing the identified tasks. This element demanded an extra effort to work in liaison with the different departments of the Ministry and also to manage changes.

\subsection{Results of the Project}

TOPOS project started in January 2012 with the development of a business case approved by the ICT Department board. After the business case approval, a number of workshops were conducted during this month to identify its initial scope and to 
Table 37.1 Initialforecast velocity

\begin{tabular}{lll}
\hline Available hours & Estimated initial velocity & Hours per story point \\
\hline 137 & 7 & 20 \\
\hline
\end{tabular}

Table 37.2 Summary of initial project plan

\begin{tabular}{llll}
\hline Magnitude & Value & Uncertainty $( \pm 25 \%)$ & Initial forecast \\
\hline Total story points & 70 & 18 & $70 \pm 18$ \\
Velocity & 7 & 2 & $7 \pm 2$ \\
Number of iterations & 10 & 3 & $10 \pm 3$ \\
Sprint length & 30 days & N/A & 30 days \\
Project length & 300 days & 90 days & $300 \pm 90$ days \\
Hours per Sprint & $137 \mathrm{~h}$ & $34 \mathrm{~h}$ & $137 \pm 34 \mathrm{~h}$ \\
Hours per project & $1,370 \mathrm{~h}$ & $340 \mathrm{~h}$ & $1,370 \pm 340 \mathrm{~h}$ \\
Cost per iteration & $€ 3,584.30$ & $€ 887.01$ & $€ 3,584.30 \pm 887.01$ \\
Total project cost & $€ 35,843.00$ & $€ 8,870.10$ & $€ 35,843.00 \pm 8,870.10$ \\
\hline
\end{tabular}

draft an initial project plan. An initial Product Backlog was created containing 21 user stories, with a total value of 70 story points, as a result of these workshops. The number and value of the stories varied during the project due to different aspects, such as new user's needs or stories re-estimation after the clarification of uncertainties. The Product Backlog included 23 user stories with a value of 64 story points at the end of the project. With the initial Product Backlog, the team established a Sprint length of 30 days, which would be stable during the project. Then, due to the lack of historical data, the team forecasted its velocity, estimating it in 7 story points and 137 initial available hours. Table 37.1 summarizes these estimations:

Based on these initial forecast s, a project plan, which included an original cost estimation founded on story points, was developed by means of the techniques proposed by Mike Cohn [13], obtaining $€ 25.90$ as the average cost per hour. It must be pointed out that, as it was developed with internal resources, all costs cited in this paper are estimations to manage the project and not real expenditures. An uncertainty percentage was added to these estimates according to McConnell's "cone of uncertainty" [23]. Table 37.2 summarizes the initial project plan:

The usual Scrum Sprint-based cycle started after the approval of the initial project plan. Each Sprint began with a Sprint planning meeting, where, depending on the available work capacity and the business priorities, the team selected the user stories that should run during the Sprint and committed to them. Moreover, each story was divided into tasks and each task was estimated. Finally, a Sprint Backlog was developed as a result of this meeting.

This artifact was the main management tool during the Sprint. Both Product and Sprint Backlog were created using Excel Spreadsheets and shared using a network folder. Before the start of the Sprint, all identified tasks were uploaded in a ticketing tool called Redmine. The team members should take responsibility for the different tasks during the Sprint upon their completion. Besides, the work performed and a 
Table 37.3 Project results against project estimations

\begin{tabular}{lll}
\hline Magnitude & Initial forecast & Final value \\
\hline Total story points & $70 \pm 18$ & 64 \\
Velocity & $7 \pm 2$ & 5.8 \\
Number of iterations & $10 \pm 3$ & 11 \\
Sprint length & 30 days & 30 days \\
Project length & $300 \pm 90$ days & 330 days \\
Hours per Sprint & $137 \pm 34 \mathrm{~h}$ & 67.72 \\
Hours per project & $1,370 \pm 340 \mathrm{~h}$ & 744.94 \\
Cost per iteration & $€ 3,584.30 \pm 887.01$ & $€ 1,777.74$ \\
Total project cost & $€ 35,843.00 \pm 8,870.10$ & $€ 19,293.95$ \\
\hline
\end{tabular}

daily estimation on the remaining work was included in the tool on a daily basis. The Scrum Master was in charge of creating a Sprint burndown chart every day and distributing it among the team members. Then, the team worked on the identified tasks during the Sprint, using this Sprint burndown chart [13] as a tool to track the remaining work in order to accomplish the committed work. It is worth mentioning that, the team was not physically placed, thus, it was not possible to hold the Daily Scrum meeting to coordinate the work. However, some electronic tools were used to be in contact such as the mentioned ticketing tool, wikis, e- mails and instant messaging tools. During each Sprint, $10 \%$ of the available time was spent to revisit the Product Backlog intending to update estimations or priorities, for instance, in what is known as "Product Backlog Grooming" [16].

The team met twice after each Sprint: the first time for a Sprint Review, to present the results of each Sprint and the other for a Sprint Retrospective, to review the process itself. It must be highlighted that retrospectives were based on the principles of Agile retrospectives [24], including techniques such as Ishikawa Diagrams, Five Whys and other innovation games. The Sprint retrospective started with a general assessment of the work performed during the Sprint, giving it a score between 1 and 5. After that, a radar diagram was developed, assessing four axis of the work: team, technology, process and environment. The team selected the most and less valuable axis and brainstormed things that should be improved or maintained. Afterwards, and using dot voting, the main problems of the Sprint and the best practices are pointed out. Lastly, Ishikawa Diagrams and Five Whys were used to find the root cause of the main problems and some improvements were proposed for each root cause. These improvements were followed up during the later retrospectives to ensure the resolution of the identified root causes.

The project lasted 11 iterations, being finished in February 2013. Table 37.3 shows the final project results and compares them with the initial estimations:

As it can be observed, the real data related to the duration of the project and the amount of work to carry it out remained within the margins of the initial forecasts.

That was not the case of the cost and dedicated hours forecasts, probably because of the absence of historical data. Therefore, this initial forecast was corrected at the end of each Sprint, as it will be shown later. Figure 37.2 shows the burnup chart of the project with the finished story points against the remaining story points of the 


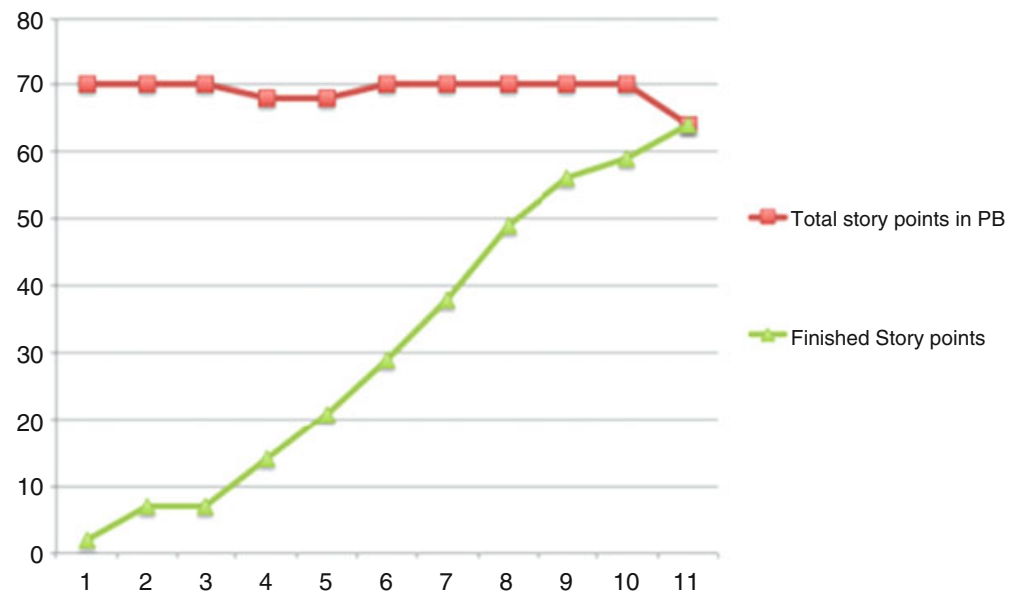

Fig. 37.2 Project burn-up chart

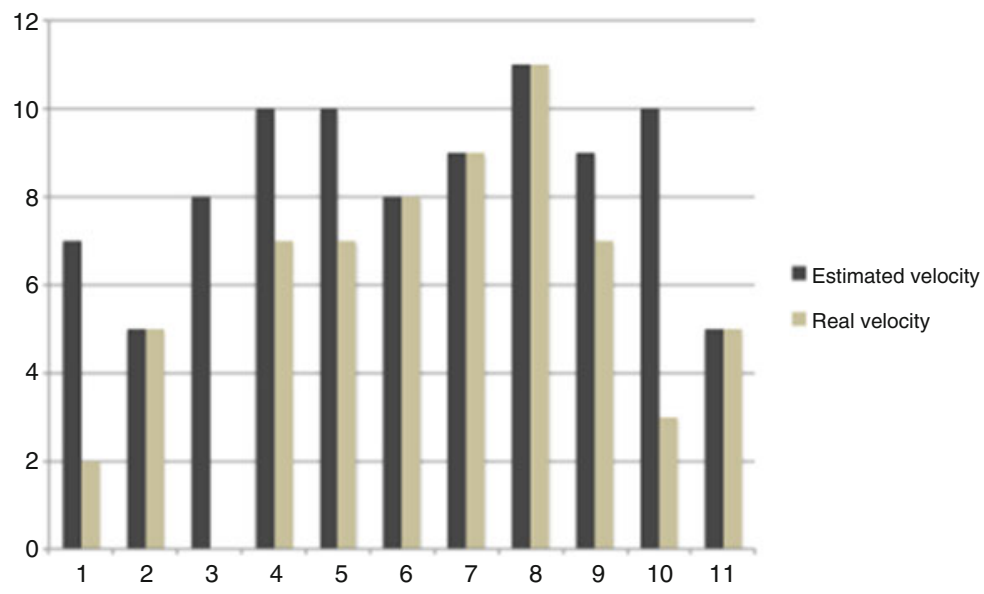

Fig. 37.3 Velocity evolution through Sprints

Product Backlog, and Fig. 37.3 represents the evolution of the real velocity against the velocity planned through the different Sprints:

As it is noticed in both figures, after the initial Sprints, the team velocity tends to stabilize and to be almost aligned with the forecasts. Table 37.4 shows the results of each Sprint, including EVM calculations:

Additionally, the cost estimation per Sprint, and therefore, the cost estimation of the project, was updated at the end of each Sprint, in order to adjust them to the real data. This also entailed, as Table 37.4 represents, the updating of the estimated hours per Sprint. Due to this fact, the estimating ability of the team improved, as both CPI 


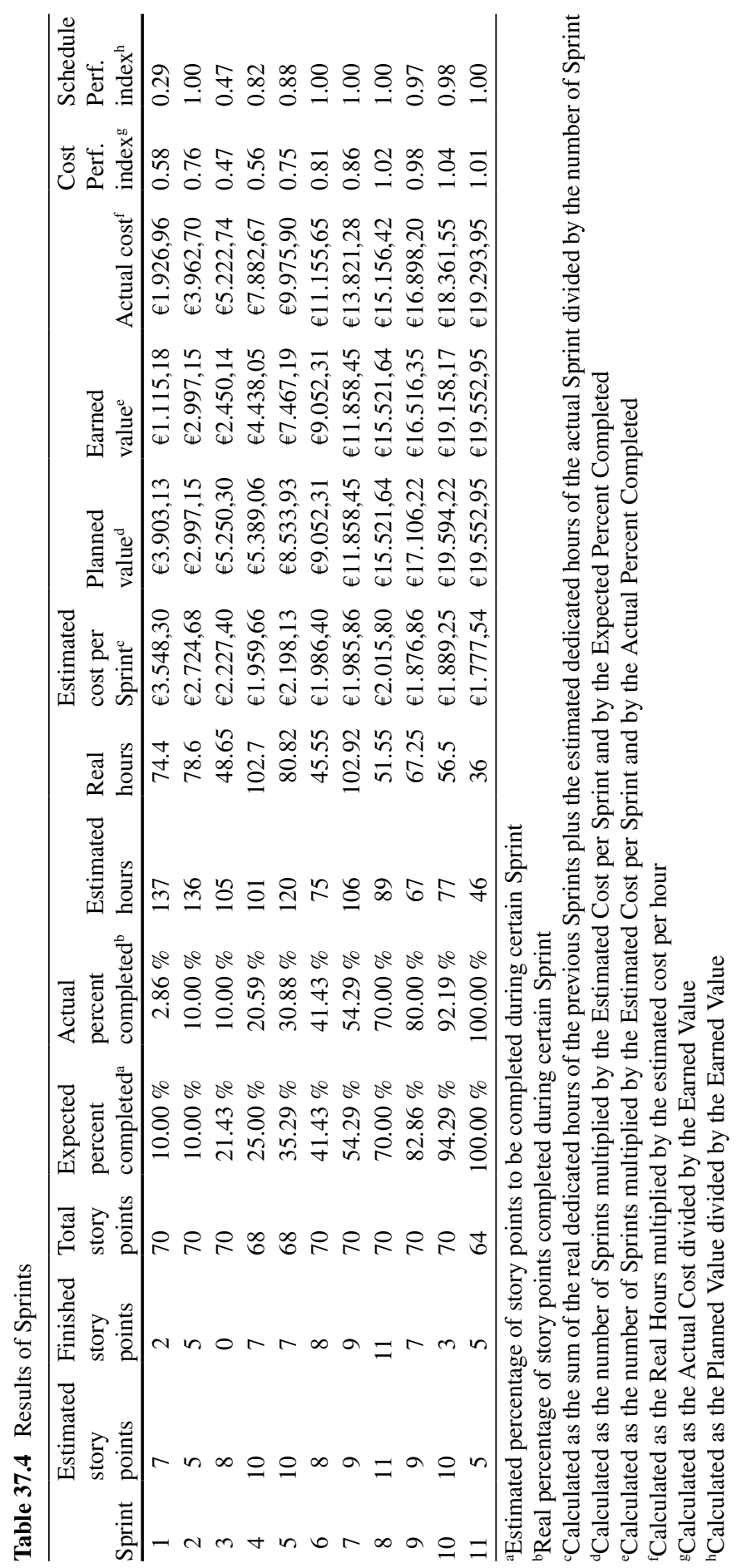


Table 37.5 Productivity metrics

\begin{tabular}{|c|c|c|c|c|c|c|c|c|}
\hline Sprint & Velocity & $\begin{array}{l}\text { Average } \\
\text { hours per } \\
\text { point } \\
\text { story }^{\mathrm{a}} \\
\end{array}$ & $\begin{array}{l}\text { Velocity } \\
\text { in hours }{ }^{b}\end{array}$ & $\begin{array}{l}\text { Work } \\
\text { capacity } \\
\text { (hours) }^{\mathrm{c}}\end{array}$ & $\begin{array}{l}\text { Focus } \\
\text { factor }^{\mathrm{d}}\end{array}$ & $\begin{array}{l}\text { Hours } \\
\text { dedicated } \\
\text { to accepted } \\
\text { work }^{\mathrm{e}} \\
\end{array}$ & $\begin{array}{l}\% \text { of } \\
\text { accepted } \\
\text { work }^{\mathrm{f}}\end{array}$ & TVI+g \\
\hline 1 & 2 & 37.20 & 74.40 & 74.4 & $100.00 \%$ & 58.9 & $79.17 \%$ & $100.00 \%$ \\
\hline 2 & 5 & 21.86 & 109.29 & 78.6 & $139.04 \%$ & 60.9 & $77.48 \%$ & $142.86 \%$ \\
\hline 3 & 0 & 28.81 & 0.00 & 48.65 & $0.00 \%$ & 0 & $0.00 \%$ & $0.00 \%$ \\
\hline 4 & 7 & 21.74 & 152.18 & 102.7 & $148.17 \%$ & 51.5 & $50.15 \%$ & $200.00 \%$ \\
\hline 5 & 7 & 18.34 & 128.39 & 80.82 & $158.86 \%$ & 40.85 & $50.54 \%$ & $166.67 \%$ \\
\hline 6 & 8 & 14.85 & 118.82 & 45.55 & $260.85 \%$ & 34.73 & $76.25 \%$ & $165.52 \%$ \\
\hline 7 & 9 & 14.04 & 126.39 & 102.92 & $122.80 \%$ & 78.16 & $75.94 \%$ & $165.79 \%$ \\
\hline 8 & 11 & 11.94 & 131.37 & 51.55 & $254.84 \%$ & 38.75 & $75.17 \%$ & $179.59 \%$ \\
\hline 9 & 7 & 11.65 & 81.56 & 67.25 & $121.27 \%$ & 41 & $60.97 \%$ & $112.50 \%$ \\
\hline 10 & 3 & 12.02 & 36.05 & 56.5 & $63.80 \%$ & 17.5 & $30.97 \%$ & $50.85 \%$ \\
\hline 11 & 5 & 11.64 & 58.20 & 36 & $161.66 \%$ & 22.25 & $61.81 \%$ & $85.94 \%$ \\
\hline
\end{tabular}

${ }^{a}$ Calculated as the sum of the dedicated working hours on the finished Sprints divided by the number of finished Sprints

${ }^{\mathrm{b} C a l c u l a t e d}$ as the sum of the completed story points in certain Sprint multiplied by the average number of hours per story point

'Total number of working hours on a certain Sprint

${ }^{d}$ Velocity in Hours divided by Work Capacity (Hours)

'Working hours dedicated to finished stories

${ }^{\mathrm{f}}$ Hours Dedicated to Accepted Work divided by Work Capacity (Hours)

${ }^{g}$ Calculated as the finished story points of the current Sprint divided by the average story points of finished Sprints

and CSPI show, becoming stable as the project advanced. Table 37.5 shows the results of the productivity metrics through the different Sprints of the project:

It is observed that, on the one hand, the average percentage of Accepted Work was almost $60 \%$, meaning that a significant amount of work during the Sprints was dedicated to deliver value to customer and, on the other hand, the average TVI+ was around $120 \%$, meaning that, on average, the team was able to deliver more story points than in the previous Sprints. Lastly, the average Focus Factor was around $140 \%$, meaning that the team tended to overestimate the efforts needed to achieve the expected results. These metrics were used during the Sprint retrospectives to guide the discussion and improve the development process.

\subsection{Lessons Learned and Future Work}

During the previous sections, an example of the application of an Agile framework, based on Scrum and including several other Agile techniques, has been presented. This framework has been relevant to estimate, plan and manage an infrastructure project developed within the Public Administration through 11 Sprints as well as to 
help the team continuously deliver value to the customers. The selected framework included the techniques to support the estimating, planning and managing efforts, as continuous processes along the project and not as an initial phase. The example shows how a long-term project can be planned using the Agile techniques, despite uncertainties, and based on the plan, be managed in an Agile way.

The team has obtained results during the project founded on an Agile framework. One important element to highlight is that this approach allows adapting the forecasts at the end of each Sprint, according to the acquired knowledge of the team. Using Agile EVM was very useful, as it improved the plan at the end of each Sprint. The team was able to learn through the project, attending to the average cost of the past Sprints and the actual forecast.

Using productivity metrics was also very useful as a guide during the Sprint retrospectives to constantly enhance the development process, pointing to certain problems in certain Sprints (for instance, whether a high percentage of working hours was not dedicated to finish and deliver stories to the customer) and to effectively know the amount of the teamwork that it is needed to deliver a value. These metrics also allow quantifying how much (or how less) the team improved during the project.

The possibility of extending and systematizing the proposed approach in order to define a general Agile framework, suitable for IT projects, at project management level, should be useful to face projects with uncertainties on requirements and needs of frequent feedback. The possibility of assessing the framework against a maturity model, as CMMI [25], will provide customers with a relevant idea of the quality of the followed development process.

Acknowledgements This research has been supported by the project QSimTest (TIN2007-67843C06_03), by the Tempros project (TIN2010-20057-C03-02), and by the project NDTQFramework (TIC-5789) of the Junta de Andaluc'a, Spain. We would also like to thank the Ministry of Culture and Sport of Junta de Andaluc'a for letting us issuing these data.

\section{References}

1. Ambler SW (2002) Lessons in agility from internet-based development. IEEE Software 19:66-73

2. Begel A, Nagappan N (2007) Usage and perceptions of agile software development in an industrial context: an exploratory study. In: Proceedings of the first international symposium on empirical software engineering and measurement ESEM '07, IEEE, Madrid, Spain, 21-27 September 2007

3. Glazer H, Dalton J, Anderson D, Konrad M, Dhrum S (2008) CMMI or agile: why not embrace both!. CMU/SEI-2008-TN-003, Pittsburgh

4. Pikkarainen $\mathrm{M}$ et al (2008) The impact of agile practices on communication in software development. Empir Software Eng 13:303-337

5. Sutherland J, Schwaber K (2011) The scrum guide: the definitive guide to scrum: the rules of the game. http://www.scrum.org/Scrum-Guides. Accessed 1 Mar 2013

6. Huang PM, Darrin AG, Knuth AA (2012) Agile hardware and software system engineering for innovation. In: Proceeding of IEEE aerospace conference 2012, Big Sky, MT, USA, 3-10 March 2012, IEEE 
7. Debois P (2008) Agile infrastructure and operations: how infra-gile are you? In: Proceeding of agile conference 2008, AGILE'08, Toronto, Canada, 4-8 November

8. Allen JN, Abdel-Aty-Zohdy HS, Ewing RL (2009) Agile hardware development with rapid hardware definition language. In: IEEE international conference on electro/information technology, 2009. EIT '09, 7-9 June 2009, pp 383-388

9. Beck K et al (2001) Manifesto for agile software development. http://www.agilemanifesto.org. Accessed 1 Mar 2013

10. Schwaber K (1995) Scrum development process. In: Proceedings of the 10th annual ACM Conference on object oriented programming systems, languages and applications, OOPSLA'95, Austin, TX, USA, 15-19 October 1995. ACM

11. Beck K, Andres C (2004) Extreme programming explained: embrace change, 2nd edn. Addison-Wesley, Boston

12. Cohn M (2004) User stories applied: for agile software development. Addison-Wesley, Boston

13. Cohn M (2005) Agile estimating and planning. Addison-Wesley, Upper Saddle River

14. Smith G, Sidky A (2009) Becoming agile in an imperfect world. Manning Publications, Greenwich

15. Highsmith J (2009) Agile project management: creating innovative products, 2nd edn. Addison-Wesley, Upper Saddle River

16. Cohn M (2009) Succeeding with agile using scrum. Addison-Wesley, Boston

17. Sulaiman T (2007) AgileEVM: measuring cost efficiency across the product lifecycle. http:// www.infoq.com/articles/agile-evm. Accessed 1 Mar 2013

18. Project Management Institute (2012) Project management book of knowledge (PMBOK) guide, 5th edn. Project Management Institute, Newtown Square

19. Downey S, Sutherland J (2012) Scrum metrics for hyperproductive teams: how they fly like fighter aircraft. In: Proceedings of 45th Hawaii international conference on system science, HICSS 2012, Maui, Hawaii, USA, 4-7 January 2012

20. Pérez García F et al (2011) Las Diferencias Regionales del Sector Público Español. Fundación BBVA, Madrid

21. European Parliament (2010) Communication from the Commission to the European Parliament, the Council, the European Economic and Social Committee and the Committee of the Regions. A digital agenda for Europe. http://eur-lex.europa.eu/LexUriServ/LexUriServ. do?uri=COM:2010:0245:FIN:EN:HTML. Accessed 1 Mar 2013

22. Spanish Parliament (2007) Law 11/2007, 22th June, on citizens electronic access to public services. http://www.boe.es/boe/dias/2007/06/23/pdfs/A27150-27166.pdf. Accessed 1 Mar 2013

23. McConnell S (2009) Software projects survival guide. Microsoft, Redmond

24. Derby E, Larsen D (2006) Agile retrospectives. Making good teams great. The Pragmatic Bookshelf, Dallas

25. Torrecilla-Salinas CJ, Escalona MJ, Mejías M (2012) A scrum-based approach to CMMI maturity level 2 in web development environments. In: Proceeding of international conference on information integration and web-based applications \& services 2012, iiWAS'12, Bali, Indonesia, 3-5 December 2012. ACM 